\title{
AKARI MID- TO FAR-INFRARED OBSERVATIONS OF DIFFUSE GALACTIC EMISSION
}

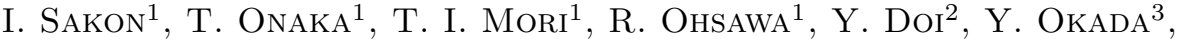 \\ H. KAnedA ${ }^{4}$, AND T. OOTSUbO \\ ${ }^{1}$ Department of Astronomy, Graduate School of Science, University of Tokyo, Japan \\ ${ }^{2}$ Department of Earth Science and Astronomy, Graduate School of Arts and Sciences, University of Tokyo, Japan \\ ${ }^{3}$ Universität zu Köln, I. Physikalisches Institut, Germany \\ ${ }^{4}$ Graduate School of Science, Nagoya University, Japan \\ ${ }^{5}$ Astronomical Institute, Graduate School of Science, Tohoku University, Japan \\ E-mail: isakon@astron.s.u-tokyo.ac.jp \\ (Received July 01, 2012; Accepted August 12, 2012)
}

\begin{abstract}
We have collected dozens of mid-infrared spectra showing UIR bands from diffuse Galactic emitting regions with the AKARI's Infrared Camera (IRC) onboard AKARI, as part of the ISMGN Mission Program. The datasets cover various directions in the inner Galactic Plane $(|l|<70 \mathrm{deg})$, in the outer Galactic Plane $(|l|>70 \mathrm{deg})$, and in the off-Plane $(|b|>2 \mathrm{deg})$. The variations in the UIR band ratios are examined in terms of the radiation environments judged from the far-infrared (50-170 $\mu \mathrm{m}$ ) spectral energy distribution (SED) made with AKARI/FIS All Sky Survey data at each slit position where mid-IR spectra were obtained. We have found that the band ratios of 6.2 $\mu \mathrm{m} / 11.2 \mu \mathrm{m}$ and $7.7 \mu \mathrm{m} / 11.2 \mu \mathrm{m}$ toward the inner Galaxy are systematically higher than those toward the outer Galaxy and off the Galactic plane. Likely causes of the variations in properties of UIR bands in diffuse emission on a Galactic scale are discussed in this paper.
\end{abstract}

key words: dust, extinction; infrared: ISM; ISM: lines and bands

\section{INTRODUCTION}

Unidentified Infrared (UIR) bands have been observed not only in the infrared spectra of celestial objects (see Tokunaga, 1997 for a review) but also in the diffuse Galactic emission (Onaka et al., 1996; Mattila et al., 1996). Although the exact nature of their carriers is not fully understood, the UIR bands appear to be ubiquitous throughout the interstellar medium and are believed to predominatly be made up of polycyclic aromatic hydrocarbons (PAHs; Duley \& Williams, 1981; Allamandola et al., 1989) and PAH-like molecular groups (e.g., amorphous organic solids with a mixed aromatic-aliphatic structure; Kwok \& Zhang, 2011). They are supposed to be formed mainly around the evolved late-type stars and to be physically and chemically modified during their ejection into interstellar space. So far, several attempts to investigate differences in the properties of the UIR bands in the diffuse Galactic emission on a Galactic scale have been made, which have aimed to demonstrate the evolution scenario of these carriers within the Milky Way. Kahanpää et al. (2003) have presented ISOPHT-S spectra of diffuse emission between $5.5-12 \mu \mathrm{m}$ at 29 positions on the Galactic plane for $|l|<60 \mathrm{deg}$ and have found no systematic variations in the relative band strengths among their datasets. We note, however, that their analysis did not cover the outer Galactic plane, where interstellar conditions are expected to be different from those in the inner Galactic plane, in particular the average interstellar radiation field strengths, metallicity etc. Sakon et al. (2004) have made a first comparison of the properties of the UIR bands in diffuse Galactic emis- 
TABLE 1.

A List of Datasets

\begin{tabular}{cccc}
\hline \hline Direction & Pointing ID & Gal. Coord. $(l, b)$ & Classification \\
\hline CAR-057 & 1400225 & $(305.5049,-0.5049)$ & inner GP \\
CAR-057 & 1400229 & $(305.5032,-0.0028)$ & inner GP \\
CAR-057 & 1400215 & $(306.5040,-0.0020)$ & inner GP \\
CRU-048 & 1400247 & $(312.0103,-0.5128)$ & inner GP \\
CRU-048 & 1400251 & $(312.5028,-0.5020)$ & inner GP \\
CRU-048 & 1400257 & $(311.5024,-0.0032)$ & inner GP \\
CRU-048 & 1400259 & $(311.5032,0.4972)$ & inner GP \\
CRU-048 & 1400261 & $(312.0024,0.4976)$ & inner GP \\
CRU+032 & 1401033 & $(35.4031,-1.0056)$ & inner GP \\
CRU+032 & 1401027 & $(32.8371,0.7203)$ & inner GP \\
CAR-079 & 1402304 & $(279.9988,-1.5065)$ & outer GP \\
CAR-079 & 1400197 & $(281.0006,-1.0090)$ & outer GP \\
CAR-079 & 1400201 & $(280.0052,-1.0007)$ & outer GP \\
CAR-079 & 1402301 & $(281.5022,-1.0024)$ & outer GP \\
CAR-079 & 1400203 & $(280.0035,-0.5065)$ & outer GP \\
PER+070 & 1402370 & $(71.0036,-1.0015)$ & outer GP \\
PER+070 & 1402369 & $(71.5059,-1.0019)$ & outer GP \\
PER+070 & 1401017 & $(70.5041,-0.5000)$ & outer GP \\
PER+070 & 1402365 & $(71.5238,-0.3946)$ & outer GP \\
PER+109 & 1402389 & $(108.4011,0.5003)$ & outer GP \\
CAR-057 & 1400231 & $(306.0051,-2.4997)$ & off-Plane \\
\hline
\end{tabular}

sion between the inner Galaxy and the outer Galaxy based on the analysis of data collected with the MidInfrared Spectrometer (MIRS) on the Infrared Telescope in Space (IRTS; Murakami et al., 1996). They reported that the band ratios for $6.2 \mu \mathrm{m} / 11.2 \mu \mathrm{m}$ and $7.7 \mu \mathrm{m} / 11.2 \mu \mathrm{m}$ were systematically larger in the inner Galaxy than in the outer Galaxy. The difference in the ionisation fraction of the carriers due to the difference in average radiation field strengths between the inner and outer Galaxy is proposed as a plausible explanation for this, but definite conclusion has not yet been given only from their datasets. In this paper, we focus on the variation in the properties of the UIR bands in the diffuse Galactic emission on a Galactic scale based on observations with the AKARI Infrared Camera (IRC; Onaka et al., 2007) and examine whether it can be explained by the difference in radiation field properties constrained from the Far-Infrared Spectral Energy Distribution (SED) analyses using AKARI Far-Infrared All Sky Survey Data (Doi et al., 2009).

\section{OBSERVATION}

The mid-infrared Spectra of diffuse Galactic emission were collected with AKARI/IRC as part of the AKARI Mission Program ISMGN (Kaneda et al., 2009). The center position of the slit for each pointed observation was defined by a comparison between the N3 image, which is taken during each pointed observation, and the 2MASS data. The data reduction procedures ba-

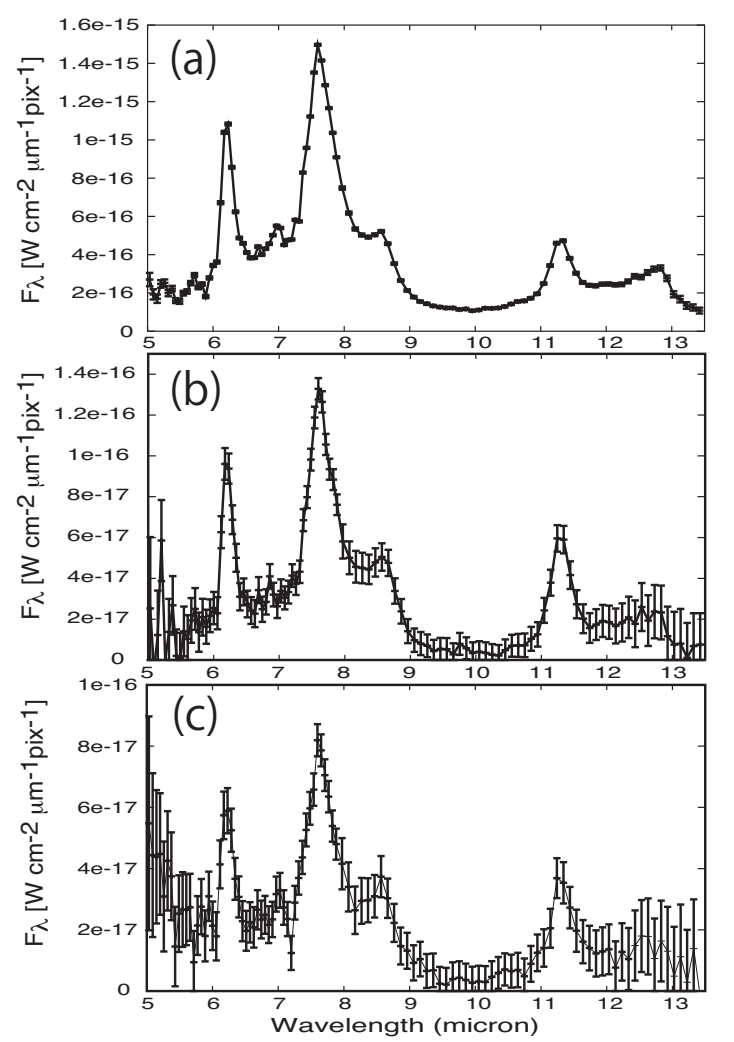

Fig. 1. The resultant AKARI/IRC MIR-S spectra (a) collected at $(l, b)=(311.5032,0.4972)$ (Pointing ID. 1400259) as a representative spectrum of the inner Galactic Plane, (b) collected at $(l, b)=(280.0035,-0.5065)$ (Pointing ID. 1400203) as a representative spectrum of the outer Galactic Plane, (c) collected at $(l, b)=(306.0051,-2.4997)$ (Pointing ID. 1400231) as a representative spectrum of the off-Plane. The zodiacal light has been subtracted from each spectrum by choosing other observation data collected at similar ecliptic latitude $\beta$ and with a Galactic longitude $|b|$ higher than $30 \mathrm{deg}$ from the archive. Extinction has not been corrected for in these plots.

sically follow those described by Sakon et al. (2007; 2008). Ten pointed observations were assigned to obtain the spectra of the diffuse Galactic emission in the inner Galactic plane (within the $8.5 \mathrm{kpc}$ galactocentric circle), another ten pointed observations were made to obtain those in the outer Galactic plane (outside the $8.5 \mathrm{kpc}$ galactocentric solar circle), and one pointed observation to obtain an off-Plane reference $\left(|b| \sim 2.5^{\circ}\right)$ (see Table 1). 


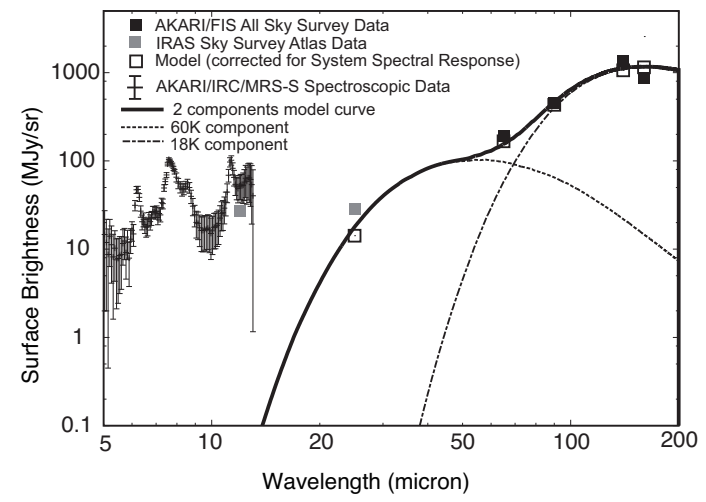

Fig. 2. An example of a SED made with AKARI FIS data at $(l, b)=(32.8371,0.7203)$ (i.e., Pointing ID. 1401027). The IRAS Sky Survey Atlas (ISSA) $12 \mu \mathrm{m}$ and $25 \mu \mathrm{m}$ band data calibrated with COBE/DIRBE Zodi-Subtracted Mission Average (ZSMA) data to correct for the zero level uncertainty and the flux scale (Wheelock et al., 1994) are also used for the fit. The FIS data are shown with black squares and the ISSA data with gray squares. The best-fit two components model curve is shown with solid line. $18 \mathrm{~K}$ and $60 \mathrm{~K}$ components are also shown with long dashed short dashed line and broken line, respectively.

\section{Galactic-Scale Variations of UIR Bands}

The mid-infrared spectra of diffuse Galactic emission obtained with AKARI/IRC are dominated by UIR band features (see Fig. 1). In order to correct for extinction effects to the UIR band strengths, empirical relations of $\tau_{6.2 \mu m}=4.8 \tau_{100 \mu m}, \tau_{7.7 \mu m}=3.8 \tau_{100 \mu m}$, $\tau_{8.6 \mu \mathrm{m}}=6.1 \tau_{100 \mu \mathrm{m}}$, and $\tau_{11.2 \mu \mathrm{m}}=4.3 \tau_{100 \mu \mathrm{m}}$ (Sakon et al., 2004) were adopted. The intrinsic UIR band ratios obtained from the spectrum at each position are summarised in Table 2 . We find that the average values of the UIR $6.2 \mu \mathrm{m} / 11.2 \mu \mathrm{m}$ and UIR7.7 $\mu \mathrm{m} / 11.2$ $\mu \mathrm{m}$ ratios in the inner Galactic Plane $(1.5 \pm 0.3$ and $3.5 \pm 0.5)$ are systematically larger than those observed toward the outer Galactic Plane (1.1 \pm 0.3 and $2.6 \pm 0.5)$ and off the Galactic Plane $(0.9 \pm 0.3$ and $2.9 \pm 0.8)$. This is consistent with the results obtained by our previous IRTS/MIRS observation (Sakon et al., 2004). Since the ionisation of PAHs is balanced by the photo-ionisation and recombination of ambient electrons, PAHs in the photo-dissociation (PDR) region tend to be positively charged. Therefore, the higher UIR $6.2 \mu \mathrm{m} / 11.2 \mu \mathrm{m}$ and UIR $7.7 \mu \mathrm{m} / 11.2 \mu \mathrm{m}$ ratios in the inner Galactic Plane should indicate more dominant contributions of $\mathrm{PDR}$ regions within a beam in the line of sight in the inner Galactic Plane than in the outer Galactic Plane and
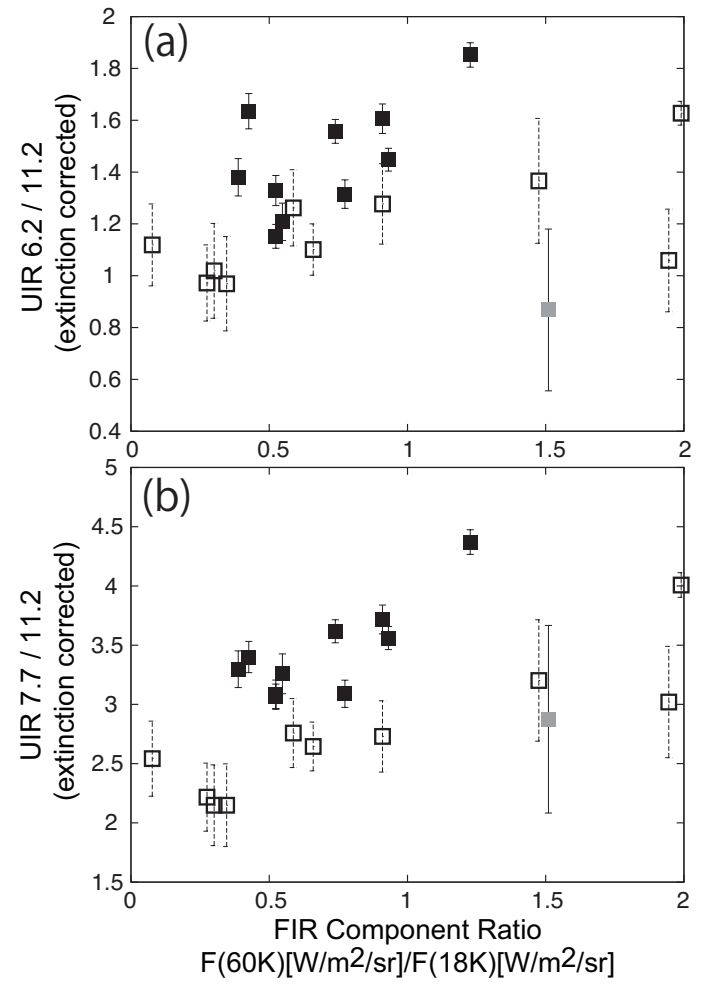

Fig. 3. Plots of $6.2 \mu \mathrm{m} / 11.2 \mu \mathrm{m}$ and $7.7 \mu \mathrm{m} / 11.2$ $\mu \mathrm{m}$ as a function of the $60 \mathrm{~K}$ to $18 \mathrm{~K}$ components ratio, $F(60 \mathrm{~K}) / F(18 \mathrm{~K})$. The solid black squares correspond to the inner Galaxy data, the open black squares correspond to the outer Galaxy data, and the gray solid square corresponds to the off-Plane data.

in the off-Plane. Since the properties of the interstellar radiation field (ISRF) cannot be constrained only from the MIR datasets, however, we used the AKARI FIR All Sky Survey data (Doi et al., 2009) to characterize the ISRF properties at the slit position.

The mid- to far-infrared SED constructed from the AKARI FIS All Sky Survey data and the ISSA 25 $\mu \mathrm{m}$ band data are fitted with a two temperature emission model (see Fig. 2). The temperatures of these components are fixed to $18 \mathrm{~K}$ and $60 \mathrm{~K}$. The $18 \mathrm{~K}$ component represents the dust emission illuminated by ISRF that is as strong as that of our vicinity ( $U \sim 1$; Mathis et al., 1983), while $60 \mathrm{~K}$ component represents the dust emission illuminated by ISRF that is 1,000 times stronger than that of our vicinity $(U \sim 1,000)$. Therefore, the total flux ratio of $60 \mathrm{~K}$ to $18 \mathrm{~K}$ components, $F(60 \mathrm{~K}) / F(18 \mathrm{~K})$, should indicate the fraction of PDR-like regions within a beam in the line of sight. In Fig. 3, the UIR $6.2 \mu \mathrm{m} / 11.2$ $\mu \mathrm{m}$ and UIR $7.7 \mu \mathrm{m} / 11.2 \mu \mathrm{m}$ are plotted against 
TABLE 2 .

Intrinsic UIR Band Ratios

\begin{tabular}{cccc}
\hline \hline Direction & ID & UIR6.2/11.2 & UIR 7.7/11.2 \\
\hline CAR-057 & 1400225 & $1.380 \pm 0.072$ & $3.297 \pm 0.155$ \\
CAR-057 & 1400229 & $1.606 \pm 0.057$ & $3.717 \pm 0.122$ \\
CAR-057 & 1400215 & $1.635 \pm 0.068$ & $3.400 \pm 0.132$ \\
CRU-048 & 1400247 & $1.329 \pm 0.058$ & $3.085 \pm 0.121$ \\
CRU-048 & 1400251 & $1.315 \pm 0.055$ & $3.090 \pm 0.115$ \\
CRU-048 & 1400257 & $1.557 \pm 0.046$ & $3.617 \pm 0.097$ \\
CRU-048 & 1400259 & $1.852 \pm 0.047$ & $4.370 \pm 0.104$ \\
CRU-048 & 1400261 & $1.448 \pm 0.044$ & $3.560 \pm 0.098$ \\
CRU+032 & 1401033 & $1.208 \pm 0.072$ & $3.258 \pm 0.168$ \\
CRU+032 & 1401027 & $1.152 \pm 0.046$ & $3.065 \pm 0.106$ \\
CAR-079 & 1402304 & $0.972 \pm 0.147$ & $2.217 \pm 0.287$ \\
CAR-079 & 1400197 & $1.262 \pm 0.147$ & $2.758 \pm 0.291$ \\
CAR-079 & 1400201 & $0.969 \pm 0.182$ & $2.149 \pm 0.349$ \\
CAR-079 & 1402301 & $1.101 \pm 0.099$ & $2.645 \pm 0.206$ \\
CAR-079 & 1400203 & $1.019 \pm 0.183$ & $2.149 \pm 0.341$ \\
PER+070 & 1402307 & $1.059 \pm 0.198$ & $3.020 \pm 0.470$ \\
PER+070 & 1402369 & $1.366 \pm 0.241$ & $3.202 \pm 0.512$ \\
PER+070 & 1401017 & $1.277 \pm 0.155$ & $2.730 \pm 0.301$ \\
PER+070 & 1402365 & $1.627 \pm 0.046$ & $4.008 \pm 0.104$ \\
PER+109 & 1402389 & $1.119 \pm 0.158$ & $2.542 \pm 0.317$ \\
CAR-057 & 1400231 & $0.868 \pm 0.312$ & $2.875 \pm 0.792$ \\
\hline
\end{tabular}

$F(60 \mathrm{~K}) / F(18 \mathrm{~K})$. Within the same category (inner Galactic Plane/outer Galactic Plane), the ratios of UIR $6.2 \mu \mathrm{m} / 11.2 \mu \mathrm{m}$ and $7.7 \mu \mathrm{m} / 11.2 \mu \mathrm{m}$ increase as the fraction of $F(60 \mathrm{~K}) / F(18 \mathrm{~K})$ increases. This can be reasonably interpreted by the trend that the contribution of positively charged PAHs increase as the fraction of PDR-like regions within a beam becomes high. However, systematic offsets among the inner Galactic Plane, the outer Galactic Plane, and the off-Plane datasets are recognized in those plots. The present results imply that the variations in the UIR $6.2 \mu \mathrm{m} / 11.2 \mu \mathrm{m}$ and UIR $7.7 \mu \mathrm{m} / 11.2 \mu \mathrm{m}$ among the inner Galactic Plane, the outer Galactic Plane, and the off-Plane cannot solely be interpreted by the difference in ISRF properties. Further investigations are needed to understand the properties of UIR bands in the diffuse Galactic emission on a Galactic scale and to demonstrate the evolution scenario of the carriers within the whole Milky Way.

\section{ACKNOWLEDGEMENTS}

AKARI is a JAXA project with the participation of ESA. The authors thank all the members of the AKARI project for their continuous support. The authors are grateful to Michael Werner and Thomas L. Roellig for useful discussion. The authors thank Glenn White for useful comments to improve this paper. This work is supported in part by a Grant-in-Aid for Scientific Research from the JSPS.

\section{REFERENCES}

Allamandola, L. J., et al., 1989, Interstellar Polycyclic Aromatic Hydrocarbons - The Infrared Emission Bands, the Excitation/Emission Mechanism, and the Astrophysical Implications, ApJS, 71, 773

Doi, Y., et al., 2009, Far-Infrared All Sky Diffuse Mapping with AKARI, ASPCS, 418, 387

Duley, W. W. \& Williams, D. A., 1981, The Infrared Spectrum of Interstellar Dust - Surface Functional Groups on Carbon, MNRAS, 196, 269

Kahanpää, J., et al., 2003, Unidentified Infrared Bands in the ISM Across the Galaxy, A\&A, 405, 999

Kaneda, H., et al., 2009, AKARI Observations of the ISM in Nearby Galaxies, ASPCS, 418, 197

Kwok, S. \& Zhang, Y., 2011, Mixed AromaticAliphatic Organic Nanoparticles as Carriers of Unidentified Infrared Emission Features, Nature, 479,80

Mathis, J. S., Mezger, P. G., \& Panagia, N., 1983, Interstellar Radiation Field and Dust Temperatures in the Diffuse Interstellar Matter and in Giant Molecular Clouds, A\&A, 128, 212

Mattila, K., et al., 1996, Spectrophotometry of UIR Bands in the Diffuse Emission of the Galactic Disk, A\&A, 315, 353

Murakami, H., et al., 1996, The IRTS (Infrared Telescope in Space) Mission, PASJ, 48, 41

Onaka, T., et al., 1996, Detection of the Mid-Infrared Unidentified Bands in the Diffuse Galactic Emission by IRTS, PASJ, 48, 59

Onaka, T., et al., 2007, The Infrared Camera (IRC) for AKARI - Design and Imaging Performance, PASJ, 59S, 401

Sakon, I., et al., 2004, The Unidentified Infrared Bands in the Diffuse ISM across the Galaxy Based on the IRTS MIRS Observation, ApJ, 609, 203

Sakon, I., et al., 2007, Properties of UIR Bands in NGC 6946 Based on Mid-Infrared Imaging and Spectroscopy with Infrared Camera on Board AKARI, PASJ, 59S, 483

Sakon, I., et al., 2008, Data Reduction Techniques for Slit and Slit-Less Spectroscopy of Diffuse Emission with the Infrared Camera Onboard AKARI, Proc. of SPIE, 7010, 88

Tokunaga, A. T., 1997, A Summary of the UIR Bands, ASPCS, 124, 149

Wheelock, S. L., et al., 1994, IRAS Sky Survey Atlas Explanatory Supplement (Pasadena: JPL) 